\title{
Playing with a nanoscale see-saw
}

\author{
Working in a small group allows you to interact closely with your advisor and your colleagues, and forces \\ you to take a lot of initiative, says Huan Li.
}

$\mathrm{M}$ $y$ research is on nanooptomechanical systems (NOMS), which are photonic devices designed for studying and controlling light-matter interactions at the nanoscale. I was first introduced to NOMS in the summer of 2010 while searching for an advisor at the University of Minnesota. Holding Bachelor's and Master's degrees in Electrical Engineering from Tsinghua University, China, I was hoping to do cutting-edge research involving both my major and physics - my favourite disciplines. Nanoscience was a natural choice. Having heard about a new faculty member who would soon be joining the Department of Electrical and Computer Engineering, I began exploring his web page. Here, I learned about NOMS for the first time and fascinated by the topic I immediately wrote an e-mail to $\mathrm{Mo} \mathrm{Li}$, who, then wrapping up in Hong Tang's lab at Yale University, wrote back within minutes and asked for my CV and transcripts. He also invited me to visit him at Yale so that he could interview me there. During the two days at Yale, I had the opportunity to tour Hong Tang's labs and talk to the postdocs and graduate students in the group. This invaluable first-hand experience helped me develop a clearer understanding of the research workflow and, more importantly, the exciting prospect of this new area.

At the end of my visit, Mo shared with me the idea of fabricating a photon see-saw device, consisting of a silicon slab suspended at its middle point and in which two photonic crystal cavities are etched. Photons can ride on either or both sides of this nanoscale see-saw and play the game via optical forces. Back then, I was just starting out as a researcher so this idea became a long-term goal of my doctoral studies. Initially, I worked on several short-term projects on NOMS and graphene photonics. I certainly benefited from such diverse training and in the fall of 2012, my first paper on an all-optical NOMS switch was published in Nature Communications 3, 1091; 2012. With the knowledge of the fundamental principles in NOMS and the skills of simulation,

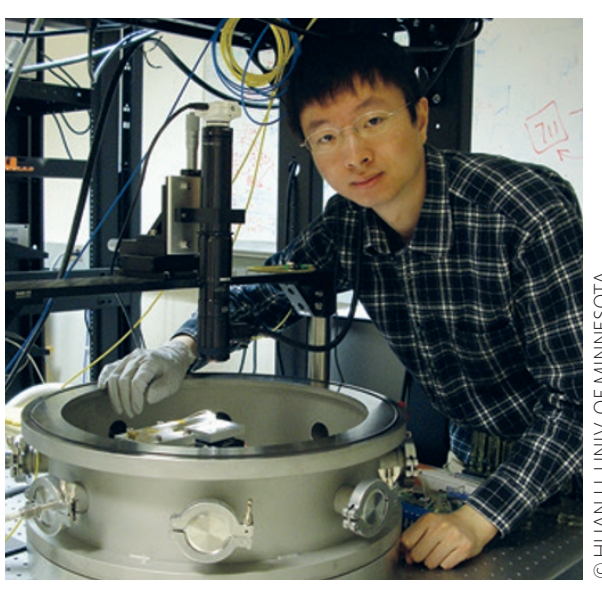

the summer of 2013, drawing on another existing design, I reduced the first design to a thin and long silicon beam with a single row of holes along it. Using my knowledge on NOMS, I further tailored the design to enhance the optical torque by two to three orders of magnitude.

Interestingly, I realized that the design now had a new feature: the beam conveniently served as a channel for the photons on both sides of the see-saw to be shuttled back and forth. Even more interestingly, the number of photons shuttled was dependent on the motion of the see-saw. So when I sent the photons into one side and let them oscillate the see-saw, I observed regulated photon packets coming out of the other side,

fabrication and experimentation acquired so far, I was ready to work on the photon see-saw project.

Fabricating the photon see-saw was a challenge because not only did we need to pattern high-quality photonic crystal cavities to effectively hold photons on the see-saw, but the delicate torsional structure also had to be suspended without collapsing. Fortunately, I frequently received help from other group members and professional staff of our cleanroom. Benefiting from such impromptu training, I was able to establish a process flow that seemed to work. By late spring 2013, after about half-year of effort, I finished the optimization and calibration of the fabrication procedure, for which the device size error was consistently within $\pm 5 \mathrm{~nm}$.

The next challenge was to optimize the design of the see-saw device, especially the photonic crystal cavities that the photons ride in. We first adopted a widely used design, taken from existing literature on photonic crystals, which consists of a silicon slab with a defective two-dimensional periodic array of holes to capture and store photons. However, I soon realized that we would need to reduce the total moment of inertia of the see-saw so that it could be easily rotated by the photons while increasing the optical torque. At the time, nobody in our group had the knowledge or experience for such cavity optimizations so I had to teach myself by reading textbooks and papers. In which agreed very well with my theoretical model. Mo and I were both excited about this, and he explained to me the broad significance of this observation, which was key to the publication of the results on page 913 of this issue. Understanding these intriguing phenomena could lead to applications in ultrasensitive measurement, quantum photonics, and future photonic computations and communications. Improvements in photonic crystal cavities and faster see-saws will further expand the possibility of these investigations.

I enjoy working in our small group because of the frequent interactions I have with my advisor and with the other group members. Also, because of the small group size (ranging between 3 and 6 people), each member typically works on different projects and is expected to be an 'all-round researcher', someone who can theorize, simulate, fabricate and experiment. Being a part of a small group allows me a rich learning experience. It affords me the opportunity to interact, learn and train in an efficient and sustained manner with my advisor and other members of the research team, and experience the entire cycle of the research workflow.

HUAN LI is about to complete his PhD at the Department of Electrical and Computer Engineering, University of Minnesota, Minneapolis, Minnesota 55455, USA. e-mail: lixx0915@umn.edu 